\title{
SIMULATING TOMORROW'S SUPPLY CHAIN TODAY
}

\author{
Randolph L. Bradley \\ The Boeing Company \\ P.O. Box 516 \\ St. Louis, MO 63166, USA
}

\author{
Jarrod Goentzel \\ Massachusetts Institute of Technology \\ 292 Main Street, Room E38-650 \\ Cambridge, MA 02139, USA
}

\begin{abstract}
Heavy industries operate equipment having a long life and rely on service parts to maintain operations. Often, stock levels for such parts are chosen to achieve fill rate goals, while supply chain performance is evaluated by speed of service. We resolve this disconnect by linking an existing discrete-event warehouse operations simulation with a new Monte Carlo demand categorization and metrics simulation. In the process, we demonstrate the potential of incorporating data on the current state of the supply chain to eliminate the simulation warm-up period and to predict future system performance against metrics targets. We show that the current stocking policy of the organization in our case study cannot achieve planned metrics and that periodic internal policies, such as budgetary approval, further degrade performance. However, a new inventory segmentation approach with continuous review can achieve targets in one year, lower inventory investment $20 \%$, and enable automated buys for certain parts.
\end{abstract}

\section{INTRODUCTION}

Heavy industries must invest in specialized service parts to maintain their equipment because, unlike automotive parts, there is often no aftermarket supplier. If parts are not on the shelf when needed, then equipment sits idle while replacements are manufactured. Stock levels for service parts are frequently optimized to reach off-the-shelf fill rate goals at minimum cost, using commercial inventory optimization tools. Performance is commonly gauged against speed of service metrics such as Order Fulfillment Lead Time (OFLT), the time from order placement to customer receipt. The OFLT metric is analogous to perfect order fulfillment, but within a delivery window that varies based upon priority. Since existing tools are neither designed to optimize to nor estimate OFLT, heavy industries cannot predict performance to their desired service metrics.

Simulation is used to analyze supply chain data from a representative industry dataset and from historical orders placed by operating organizations needing to maintain their equipment over time. When the analysis revealed that the desired metrics were not achievable, a potential intervention in the supply chain management strategy was devised. We test the hypothesis that OFLT, using a simple inventory segmentation strategy to group consumable parts by cost, can be improved through a more sophisticated strategy that groups parts by annual use, or cost times demand.

In the process, this research presents a useful technique for predicting future performance over time, given supply chain uncertainty, in order to provide guidance in (a) setting appropriate stock level goals, and (b) estimating when program performance will achieve contractual goals. The solution involves federating (building a system of systems) an existing large-scale simulation with a "bolt on" secondary simulation, demonstrating how complex legacy models can find new life by feeding data to specialized simulations. This paper offers a real world case study to demonstrate that operations in non-steady state conditions can be effectively evaluated into the future using simulation without a warm-up period. 


\section{Bradley and Goentzel}

\section{REVIEW OF THE RELEVANT LITERATURE}

Law and Kelton (2000) describe the successful use of simulation to understand large-scale systems, such as "determining ordering policies for an inventory system." Simulation is the classic approach to studying complex engineered systems. Given that simulation is the chosen technique for estimating the Order Fulfillment Lead Time metric, this literature review focuses on two areas: (1) applications of inventory optimization to maintenance supply chains, and (2) applications of discrete-event simulation to maintenance supply chains. These references describe recent technical approaches that can enable companies to achieve supply chain metrics tomorrow at lower lifecycle cost than today.

\subsection{Optimizing Stocking Policies for Service Parts}

The use of multi-echelon inventory optimization for service parts, characterized by low demand probabilities, high cost, and high priority for service characterized by "response time service levels" is described by Cohen, Kleindorfer and Lee (2006); Inventory Optimization Model A referenced in this paper uses this technique. A systems approach to setting stock levels for multi-echelon, multi-indenture equipment in fleets of commercial and military aircraft, ships, and power plants is described by Sherbrooke (2004), along with distinguishing between the treatment of consumable and reparable parts; Inventory Optimization Model B uses this technique. Fuerst (1981) describes the classic ABC inventory segmentation analysis from a practitioner's standpoint. Rossetti and Achlerkar $(2004,2009)$ discuss techniques for spare parts segmentation, presenting a summary review of the literature on multi-echelon inventory models and clustering approaches to inventory segmentation. McKinsey \& Company (2008) notes that service, inventory, and profitability improve when industry implements supply chain segmentation techniques based on product needs and demand patterns. The focus of planning departments also improves.

\subsection{Simulating Stocking Policies}

Integrating inventory optimization and simulation to analyze supply chain effectiveness is espoused by Ingalls, Cornejo, Methapatara and Sittivijan (2008), noting that, "Given a mathematically optimal solution as recommended by optimization, simulation shows how that optimal solution will perform under dynamic conditions." A modular approach to simulation in which modules are related based on process, but independent based on coding, is described by Shoaf (1983). The commercially available ExtendSim simulation language is designed to integrate simulation with the supporting data that defines the scenario (Diamond, et. al., 2010). The discrete-event warehouse simulation described in this paper closely follows the approach demonstrated by Saylor and Dailey (2010) and is based on a library of reusable supply chain modules for evaluating manufacturing schedules, performing inventory analysis, and modeling supply chains that uses ExtendSim as the foundation. Practical methods to verify and validate simulation models are discussed by Sargent (2010); this paper employs the techniques of data validity, comparison to other models, face validity, historical data validation, internal validity, parameter variability - sensitivity analysis, and predictive validation. Banks, Carson, Nelson and Nicol (2001, pp. 414-416) apply a statistical process to calculating the minimum number of repetitions of a simulation.

Skoogh, Michaloski and Bengtsson (2010) recommend automating data collection and processing to integrate supply chain simulation as a daily tool, rather than a single-purpose study; the Extract, Transform and Load (ETL) tool of this paper automates the creation of simulation datasets, based on input data contained in an inventory optimization model, representing a significant step in this direction.

Dorey (2011) provides an overview of standard and incentive contract types, proposes to characterize contract risk by using simulation to map probabilistic cost estimates to profit distributions, and recommends risk-driven contract structures to impose cost sharing and bound cost growth; the Order Fulfillment Lead Time metric discussed in this paper is an example of a speed of service metric that would be attached to a risk-driven service parts contract. 


\section{Bradley and Goentzel}

\section{METHODS AND DATA}

A forward looking analysis of a supply chain provides a competitive advantage: if you can predict the future, you can continue strategies that work and alter those that do not. By melding models though shared data, this paper demonstrates the capability to integrate inventory optimization, warehouse simulation, and metrics simulation to evaluate supply chain strategies.

Performance over time for OFLT, resulting from stock levels determined by a strategic inventory optimization model used for long range planning, is estimated by two linked simulation models as shown in Figure 1. First, a discrete-event warehouse simulation evaluates orders of varying size for parts that fail according to a Poisson distribution. Time is important because inventory levels vary based on demand for spare parts, and replenishment occurs within manufacturing lead time when parts reach their reorder point. This warehouse simulation creates a list of orders that occur over time. Second, a Monte Carlo demand categorization and metrics simulation reviews each warehouse order after using empirical probability distributions to determine order priority, order size, order type (for metrics purposes), and shipping delay. Empirical distributions for these random variables are developed using historical data. The Monte Carlo simulation determines OFLT metrics on a monthly basis into the future.

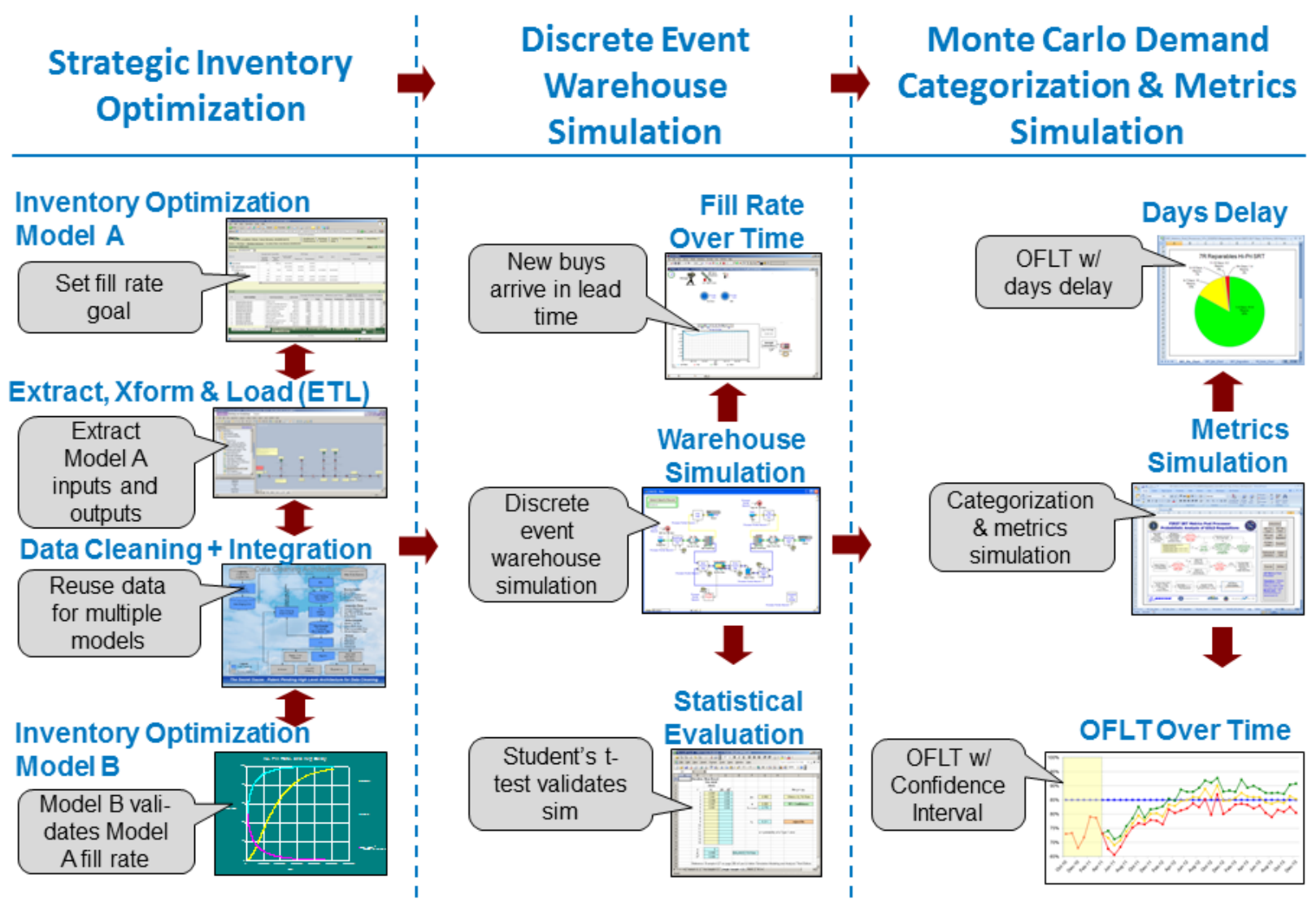

Figure 1: The methodology for simulating Order Fulfillment Lead Time involves inventory optimization, warehouse simulation, and metrics simulation

The operational context of our case study is a single warehouse, operated by a third party logistics vendor, supplying three maintenance depots that support a fleet of approximately 500 high value pieces of complex equipment, which are deployed to 20 locations and operated by two customers. Each piece of equipment operates approximately 20 hours per month. The fleet average monthly demand across the 


\section{Bradley and Goentzel}

14,000 unique consumable SKUs is 42,000 parts, and demand across the 650 unique reparable SKUs is 250 parts.

Required supply chain data include the inputs (current on hand, due-in, and backorder inventory position, demand, lead time, price, condemnation rate, and maintenance concept) and outputs (target stock level, reorder point, and reorder quantity) of a commercial inventory optimization model. Also required are historical requisition data (part number, order quantity, and date), shipping performance data (days shipping delay by requisition, priority by requisition), performance (as reported within a company), and operational data including equipment delivery schedule and future operating hours by period. After benchmarking the new predictive simulation models to an existing commercial inventory optimization model by comparing fill rate results, the new models were used to estimate OFLT over time.

Unlike most supply chain simulation models, which require an extended warm-up period and only estimate steady state conditions, this approach takes into account the current state of the supply chain, duein orders with scheduled delivery dates, and optimized stocking policies in order to estimate OFLT over time. In this manner, these simulations extend the state of the art by eliminating the warm-up period, thus providing both short-term and long-term predictions of performance to contractual metrics.

\section{SIMULATING ORDER FULFILLMENT LEAD TIME}

The authors designed the Monte Carlo demand categorization and metrics simulation to answer the question, "When will Order Fulfillment Lead Time improve to the desired level of performance?" This standalone software application uses Visual Basic for Applications (VBA) and Excel 2010 to run a Monte Carlo simulation of the following: the probability that a part requisition generated by the discrete-event warehouse simulation is categorized as "on metric", the probability a part is ordered as high or low priority, and the probabilistic shipping delay.

\subsection{Federation of Models}

These two inventory optimization models and two simulation models were joined together by linking process (the order of use) and data (inputs and outputs). This solution offers an example of how a complex systems can be evaluated through a federation of different types of models.

The objective function of each inventory optimization model, when optimizing consumable parts subject to an inventory segmentation strategy, is to minimize inventory investment for a given fill rate. The inputs to this model include the operational scenario (number of equipment, by equipment model; operating hours by month; operating locations; repair hierarchy), part level data (part number, manufacturer's code, nomenclature, price, mean operating hours between demand, condemnation rate for reparable parts, repair turnaround time for reparable parts, procurement lead time), and part and location level data (inventory on hand, due-in, and on backorder). The outputs of this model include stock level recommendations by part and by location (target stock level and reorder point).

The discrete-event warehouse simulation analyses the inputs and outputs of the inventory optimization model in order to predict fill rate over time, as orders are placed to bring low starting stock levels up to the recommended target stock levels, and excess inventory is used up, or "burned down", over time. Orders are delivered in the fixed procurement lead time of each part, and demand is variable based on the monthly equipment operating hours. Of the dozens of input and output tables of this simulation, the only table required for the next step in our overall process is the list of transactions from the operating locations (retail), to the central warehouse (wholesale).

The Monte Carlo demand categorization and metrics simulation adds probabilistic data to the discrete-event warehouse simulation results, based on empirical data for the percentage of time an order counted towards metrics (on metric), the percentage of time a part was ordered as high priority or low priority (based on history), historical shipping delays, and historical order quantities. Thus, the Monte Carlo simulation transforms the fill rate output of the discrete-event simulation into the more useful Order Fulfillment Lead Time (OFLT) metric. 


\section{Bradley and Goentzel}

\subsection{Model Formulation}

The Monte Carlo demand categorization and metrics simulation utilizes the decision variables shown in Table 1. The variable in green (conrep_bst) allows demand categorization, and is specific to the part requisition. Variables in red (metric_bst, hipri_bst, shiphi_bst, and shiplo_bst) are Monte Carlo variables which change with each iteration.

Table 1: Key parameters for the Monte Carlo demand categorization and metrics simulation

From the discrete-event warehouse simulation model, for Part $i$

Close_Date $_{i} \quad$ The date and time of that the requisition is filled from inventory in the wholesale warehouse, excluding shipping delay

Days_to_Fulfill Requisition_Date $_{i}$-Close_Date ${ }_{i}$

Needed_Q $Q_{i} \quad$ Quantity needed/quantity ordered

Released_Q_SR_No_Delay ${ }_{i} \quad$ Quantity released to fulfill the supply requisition with no delay (the order was satisfied from on hand inventory)

Requisition_ $Q_{i} \quad$ Requisition quantity, which is based on an empirical order quantity distribution, in integer parts

Requisition_Date $_{i} \quad$ The date and time of the requisition placed from retail to wholesale Supply $_{i} \quad$ Unique reference number/part number/SKU

From the Inventory Optimization Model, for Part $i$ or classification class

metric_bst $t_{i}$

Percent of requisition categorized as "on metric", where "on metric" requisitions are defined as counting towards metrics

conrep_bst

hipri_bst $t_{i}$ Repair classification/category (either consumable or reparable)

shiphi_bst class

shiplo_bst class

Percent of requisitions ordered as high priority

Empirical shipping delay distribution, in whole days, for requisitions ordered as high priority, where class is either consumable or reparable Empirical shipping delay distribution, in whole days, for requisitions ordered as low priority, where class is either consumable or reparable

This approach offers several advantages for modeling. First, the existing warehouse simulation required only minor adjustments to (a) incorporate an empirical order quantity distribution, and (b) allow for periodic resupply. Second, the new demand categorization simulation was based on only one table from the warehouse simulation, allowing (a) the entire table to be imported as an input, simplifying the link between the simulations, (b) providing traceability during model development to the exact source data from the simulation, and (c) allowing the warehouse simulation graph of cumulative fill rate, a standard model output, to be compared to analogous graph from the metrics simulation, offering an expedient validation of the basic categorization and graphing of the second simulation.

The process for simulating the key metric, Order Fulfillment Lead Time, follows the process in Figure 2, which describes the logic used to determine whether a specific order counts towards metrics, and if so, whether the metrics were achieved. The overall simulation loops over runs of the Monte Carlo demand categorization and metrics simulation and the discrete-event warehouse simulation for a set of part requisitions. This process includes the following key steps:

- Determine whether the requisition counts toward metrics based on characteristic of the order, which is specific to the part number and is highlighted in Figure 2 in green:

- What is the repair classification, which is either consumable or reparable?

- Categorize the requisition based on characteristics that change order to order and are highlighted in Figure 2 in red:

○ Is the item "on metric", in which case the order counts towards metrics?

$\circ$ What is the priority of the order? High priority indicates that the part is necessary to return equipment to service, and low priority indicates a routine reorder. 


\section{Bradley and Goentzel}

- What is the shipping delay, which historically varies order to order?

- Calculate confidence interval using Student's t-Test for the warehouse simulation runs.

- Graph charts of service metrics over time.

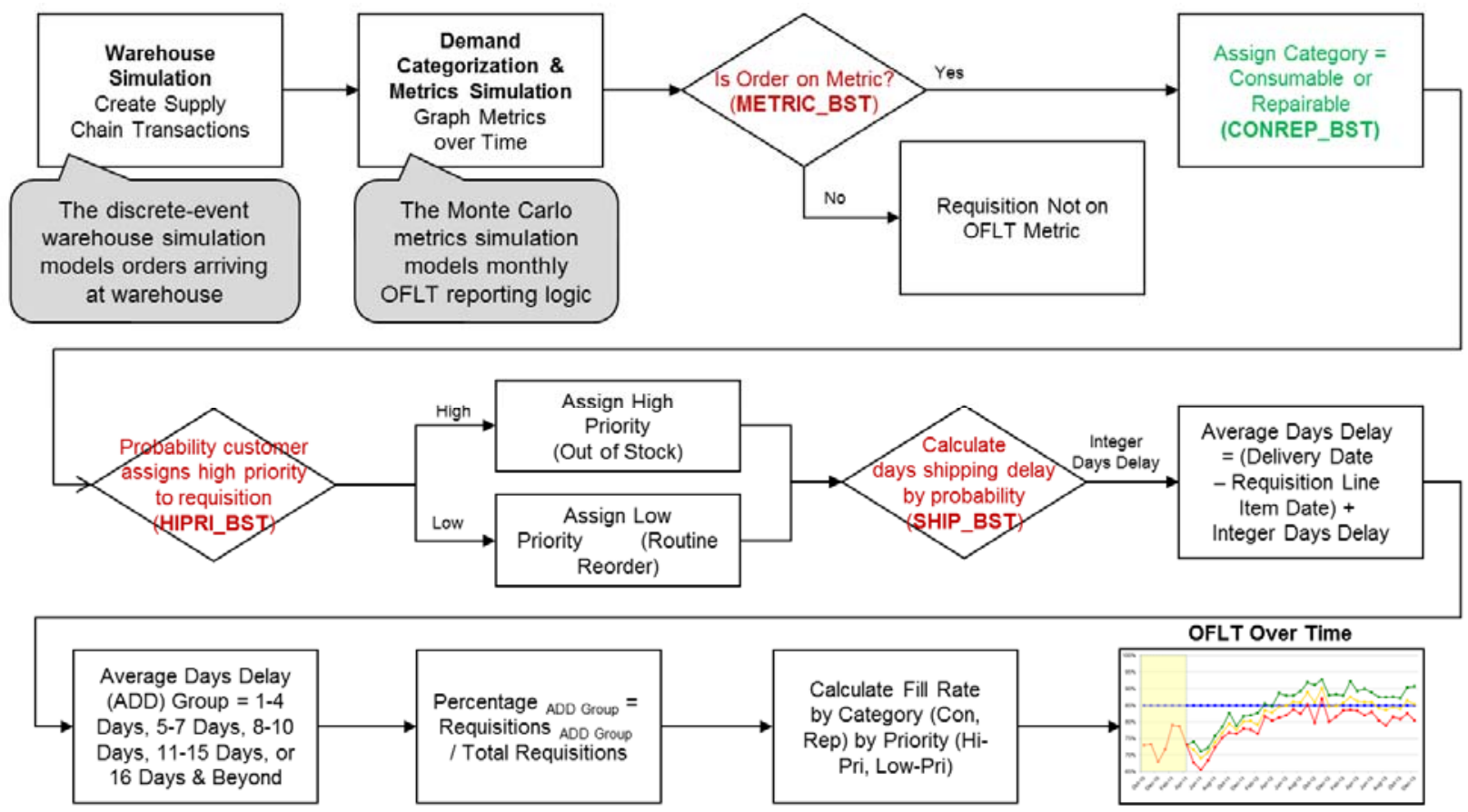

Figure 2: Methodology for calculating supply chain metrics using linked simulations

\subsection{Initialization without Warm-Up Period}

Unlike most supply chain simulations, which require an extended warm-up period and estimate only steady state conditions, these linked models take into account the existing state of the supply chain, due-in orders with scheduled delivery dates, and optimized stock levels and reorder points in order to estimate OFLT over time, as of the exact date of the data pull. By eliminating the warm-up period, these simulations extend the state of the art to provide short-term in addition to long-term predictions of performance to service metrics.

The strategy of linking a warehouse simulation of transactions with a metrics simulation for evaluation allowed for an interesting enhancement. By seeding the elapsed time period between the start of the current contract and the start of the simulation with historical transactions, it is possible to (a) graph the transition from actual (reported) metrics to simulated (predicted) metrics, and (b) estimate cumulative statistics precisely, by accounting for the actual results since the start of the contract period.

\subsection{Validation}

Substantiating the analysis encompasses both stock level validation and simulation validation. For the stock level validation, the expected fill rate from Inventory Optimization Model A was verified by running Inventory Optimization Model B in evaluation mode and comparing fill rates for both the current inventory position, and for the recommended stock levels. While it is recognized that both models have different inventory optimization engines, the hypothesis is that stocking policies from one model should yield comparable results when evaluated in the other model. This validation exercise also serves as a check against gross errors. 


\section{Bradley and Goentzel}

For the simulation validation, the target stock levels specified by Inventory Optimization Model A were evaluated in both the discrete-event warehouse simulation and the Monte Carlo demand categorization and metrics simulation. Both simulations produce results that are close to the inventory optimization, thus validating (a) the data extract, (b) the demand calculations in the different tools, (c) the ability of the target stock levels to achieve the stated fill rates.

\section{OPTIMAL NUMBER OF SIMULATION RUNS}

The optimal number of runs necessary to draw conclusions is calculated by examining the confidence interval attained progressively through the simulation. When the confidence interval either (a) converges to a steady state value, or (b) reaches an acceptable level of accuracy, the lowest number of replications has been reached (Webster 2011). These methods are described by Robinson (2004) as the graphical method and the confidence interval method.

The discrete-event warehouse simulation was executed for 21 iterations. Each iteration requires about an hour to prepare, in addition to about two hours of computer time on a workstation running Windows 7 with an Intel Core i7-950 processor and 12 gigabytes (GB) of memory. The resulting confidence intervals for OFLT are shown in Figure 3, with the confidence interval over time shown above, and select confidence intervals by month shown below.

The results using four and 21 replications produce remarkably similar results. As the lower charts in Figure 3 show, the $80 \%$ confidence interval narrows notably when moving from three to four simulations, but the marginal benefit of each additional simulation run is much less. Given the likelihood that procurement lead time will be variable (which was not simulated for this paper, but is common in industry), or that other endogenous uncertainties, which are influenced by managerial decisions, will come to pass, a $+/-2-4 \%$ confidence interval is sufficient. For this reason, four iterations of the discrete-event warehouse simulation were chosen as appropriate. The number of runs required for the Monte Carlo demand categorization and metrics simulation was evaluated in a similar manner, and determined to be twelve runs. Each run requires about 20 minutes.
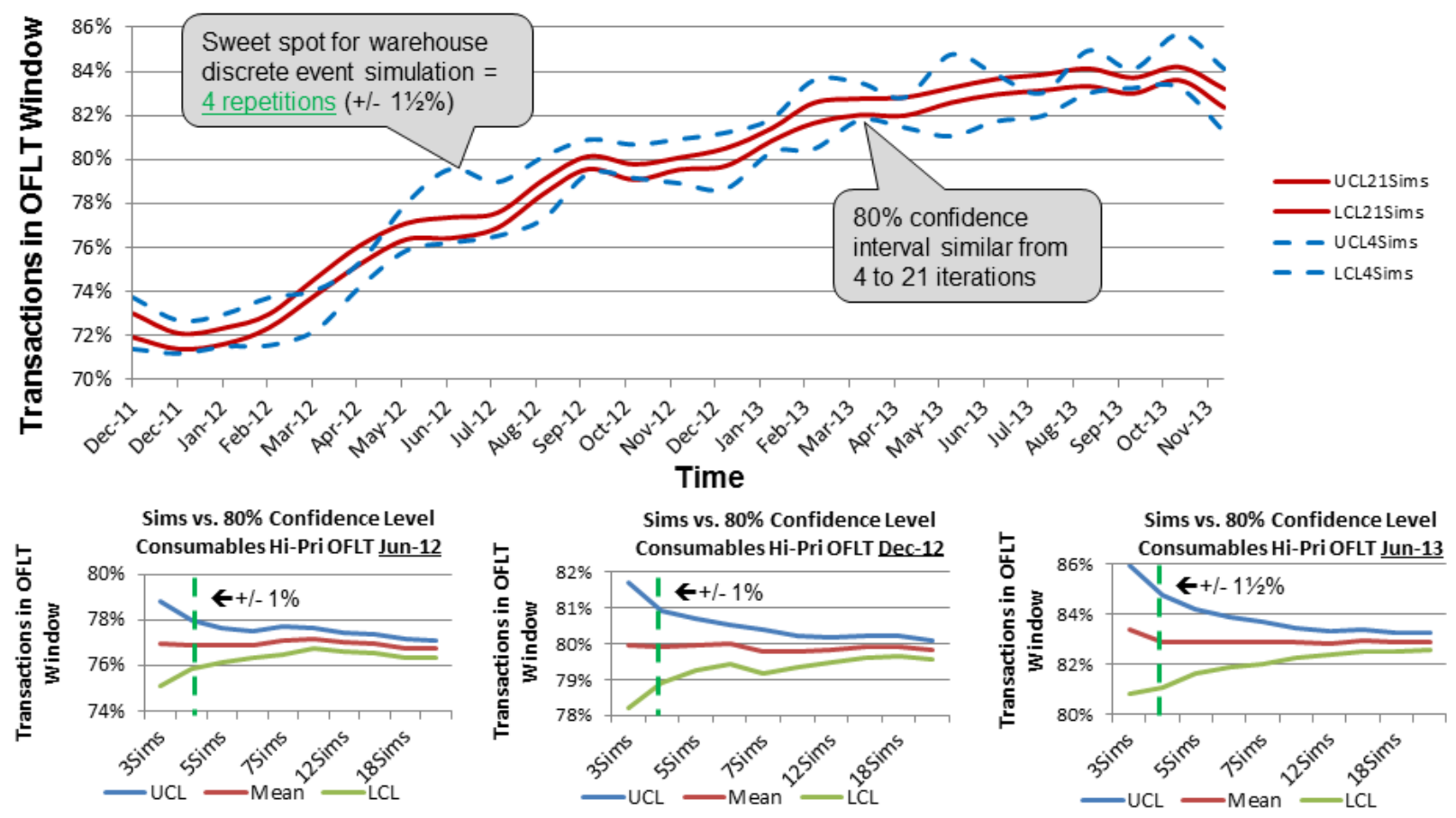

Figure 3: The sweet spot for iterations of the discrete-event warehouse simulation is four (4) runs 


\section{Bradley and Goentzel}

\section{SIMULATING INVENTORY SEGMENTATION STRATEGIES}

Consumable parts, often grouped by segment, are commonly optimized to fill rate targets. Supply chain performance is frequently gauged against a speed of service measure such as Order Fulfillment Lead Time (OFLT), the time from order placement to customer receipt. Simulation was used to evaluate inventory segmentation strategies for consumable parts, as measured by OFLT and inventory investment, by analyzing an industry representative dataset.

\subsection{Simple Strategy: Segmenting Inventory on Cost}

A simple strategy segments parts into A and B categories based on unit price, as shown in Figure 4. Each segment is optimized separately to a fill rate goal using a commercial inventory optimization model (Cohen, Kleindorfer, and Lee 1986). A business rule ensures that all parts are at least stocked to the Economic Order Quantity (EOQ) amount, ensuring a minimum 50\% fill rate by part. This simple strategy is common in heavy industry.

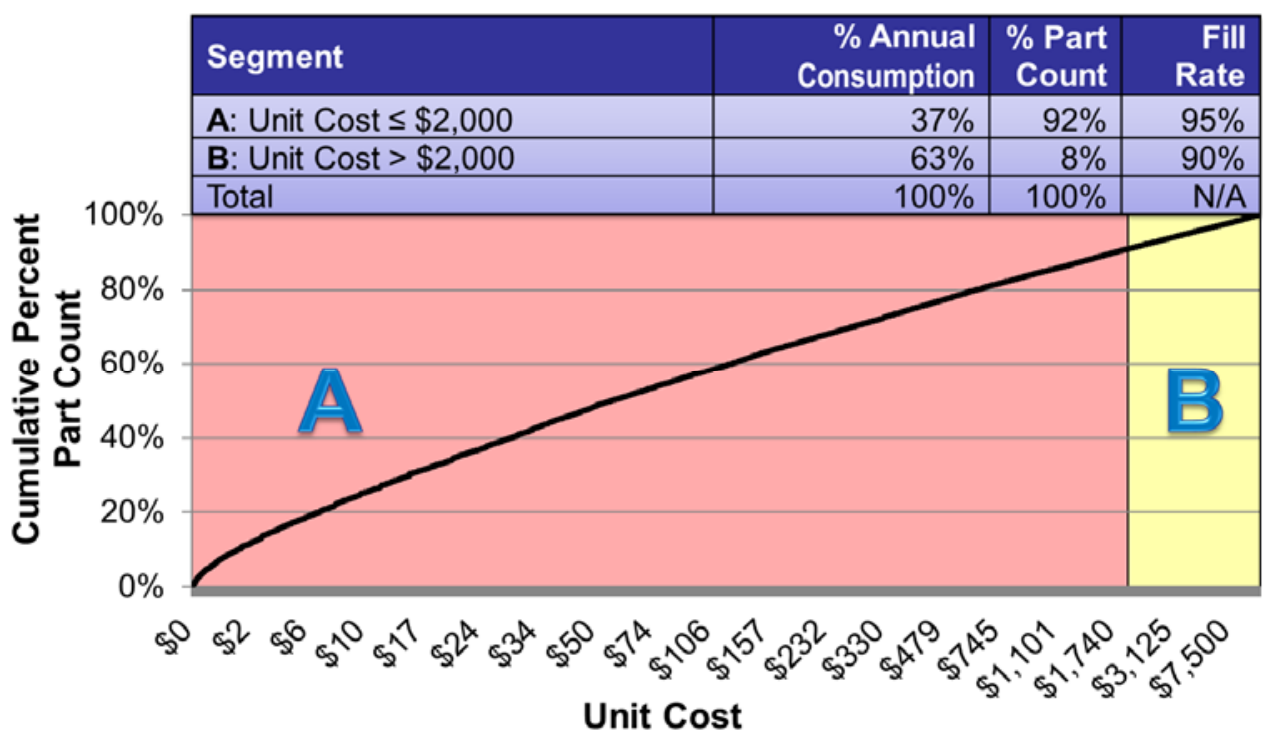

Figure 4: Simple strategy for inventory segmentation, segmenting consumable parts on cost (top), and showing ordered unit cost vs. cumulative part count (bottom).

Consumable parts for Segments A and B were ranked from lowest to highest unit price on the horizontal axis, compared to cumulative part count on the vertical axis, and graphed in Figure 4. Since an inventory optimization model uses a technique known as a "greedy heuristic" to select the lowest cost mix of spares required to achieve a given fill rate goal (Sherbrooke 2004), this means that for Segment A, $1 \varnothing$ parts are competing with $\$ 1,740$ parts on "bang for the buck" to increase fill rate. Since this strategy achieves fill rate goals without stocking many expensive parts, the business rule was added in an attempt to better balance the mix of parts.

\subsection{Sophisticated Strategy: Segmenting Inventory on Consumption}

A sophisticated strategy segments parts into five categories based on "consumption" (which is defined as the annual demand multiplied by unit price), as shown in Figure 5. Decision rules for these parts balance an asset manager's time against the benefit of in-depth analysis (Silver, Pyke, and Peterson 1998):

- Class A parts should be carefully tracked by asset managers. Instead of placing an order, could the asset manager transfer existing items to eliminate an imbalance of inventory between locations? Could the buyer reduce unit cost through competition among suppliers? 


\section{Bradley and Goentzel}

- Class B parts are in the mid-range, and should receive moderate attention, with management by exception rules flagging asset managers when close attention is warranted.

- Class $\mathbf{C}$ parts should be managed with simple decision rules. The value of an asset manager's time in researching stockouts is greater than the holding cost incurred from high fill rates. These parts should be managed electronically to target stock levels, without human intervention.

- Class D parts cost from $\$ 1$ to $\$ 25$, and should be managed with simple decision rules to achieve high fill rates.

- Class E parts cost $\$ 1$ or less, and should be managed with simple decision rules to achieve very high fill rates.

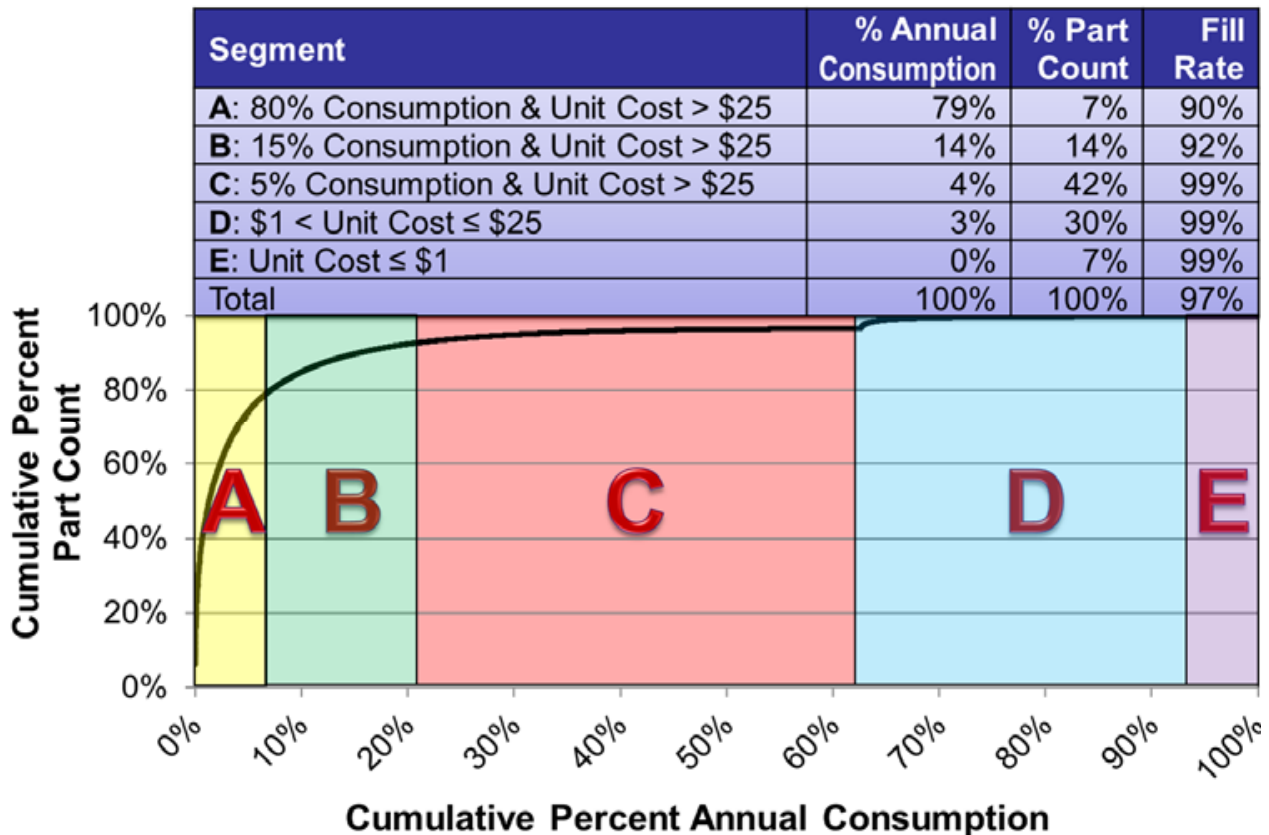

Figure 5: Sophisticated strategy for inventory segmentation, segmenting consumable parts on consumption (top), and showing annual consumption vs. cumulative part count (bottom).

\subsection{Results}

This research tests the hypothesis that the sophisticated strategy, which segments consumable parts on consumption (a combination of volume and cost), will outperform the simple strategy, which segments only on cost, when measured on both Order Fulfillment Lead Time and inventory investment. The null hypothesis -- that there is no difference between inventory segmentation strategies when measured on OFLT -- is rejected with a $95 \%$ level of confidence. At the start of the simulation, orders are placed to bring inventories for parts at or below reorder point up to the target stock level. Performance improves as these parts arrive in the warehouse after manufacturing lead time. Indeed, simulation results for high priority consumable parts indicate that by August 2013, the mean OFLT for the sophisticated strategy will achieve the desired goal, as indicated by the vertical dashed green line in Figure 6, unlike the simple strategy, which fails to meet goals.

Second, the sophisticated strategy also achieves a $20 \%$ reduction in investment compared to the simple strategy. This was unexpected, because the latter skews stock levels in favor of low unit price parts. A business rule requiring a minimum $50 \%$ fill rate by part, added in an attempt to improve the parts mix, reduced the benefits of cost optimization in the simple strategy. 


\section{Bradley and Goentzel}

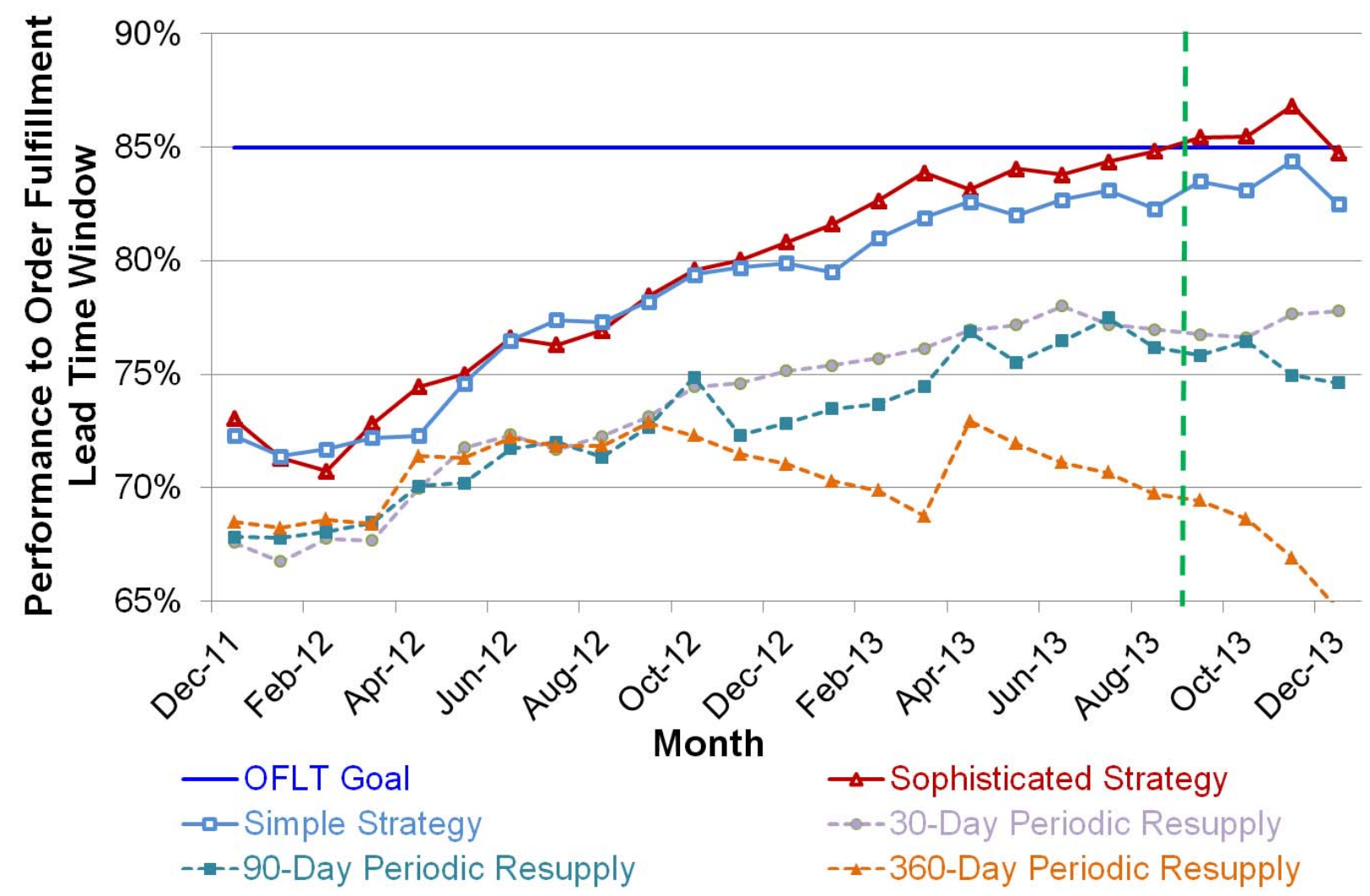

Figure 6: Stock levels for consumable parts using the sophisticated strategy outperform the simple strategy, achieving Order Fulfillment Lead Time goals by August 2013 (dashed green vertical line). Imposing 30-day, 90-day, and 360-day resupply periods on the simple strategy degrades performance over time.

\subsection{Stocking Policy}

Stocking policy, review policy, and acquisition policy go hand in hand. A continuous review stocking policy is appropriate for a flexible contract that funds purchases when stock levels fall to reorder points. A review board that meets monthly to approve orders results in a de facto periodic review policy. Similarly, quarterly or annual funding cycles result in a periodic resupply contract. In these cases, the stocking policy must be changed from continuous review (with frequent buys to stock level) to periodic review (with monthly, quarterly, or annual buys to stock level). The appropriate review period must be added to the procurement lead time. The stock levels recommended by an inventory optimization model increase as the review period increases.

The key to understanding the impact of the review period and acquisition policy is realizing their effect on OFLT metrics for the simple strategy, also shown above in Figure 6. A monthly spares requirements review board imposes a 30-day delay on placing orders, resulting in about a five percentage point penalty on OFLT, sufficient to cause an organization to continuously miss goals. The 90-day delay incurred from a quarterly acquisition policy drops performance another few percent. The 360-day delay incurred from an annual acquisition policy degrades performance over time.

\section{CONCLUSIONS}

This research provides insights for supply chain strategy and for simulation methodology. First, heavy industry can achieve supply chain service metrics, lower inventory investment, and lower asset management costs based on results from the industry representative dataset. Organizations that stock service parts can lower inventory investment in consumable parts while increasing performance on speed of ser- 


\section{Bradley and Goentzel}

vice metrics using a more sophisticated segmentation approach. In addition, organizations must be cognizant that review policy and acquisition policy impact results. Automating the reorder of parts in the C, D and $\mathrm{E}$ Segments eliminates the pipeline stock required by the review period, allowing asset managers to focus their analysis on critical A and B Segments. Authorizing flexible funding eliminates the need to add additional months of pipeline stock to compensate for funding cycles.

Second, our approach demonstrates that simulation not only can reveal whether service goals are achievable, but when, and with what confidence. Using current data to initialize simulation rather than commencing simulation at an unrealistic zero point and waiting for the model to converge to steady state eliminates the warm-up requirement and improves computational efficiency. Moreover, the simulation can then be used to predict improvements to performance over time from different supply chain strategies. Beyond predicting whether goals are attainable or not, our approach predicts when they will be attained.

Finally, by federating existing models for inventory optimization and discrete-event warehouse simulation with a new Monte Carlo metrics simulation, we demonstrate how existing models can be effectively reused and combined when evaluating complex systems. New capabilities can be created by extending legacy code with little or no modification.

\section{REFERENCES}

Banks, J., J. S. Carson II, B. L. Nelson, and D. M. Nicol. 2001. Discrete Event System Simulation 3rd ed. Upper Saddle River, NJ: Prentice Hall, Inc.

Cohen, M. A., P. R. Kleindorfer, and H. L. Lee. 1986. "Optimal Stocking Policies for Low Usage Items in Multi-Echelon Inventory Systems.” Naval Research Logistics Quarterly 33:17-38.

Diamond, B., D. Krahl, A. Nastasi, and P. Tag. 2010. "ExtendSim Advanced Techology: Integrated Simulation Database." In Proceedings of the 2010 Winter Simulation Conference, edited by B. Johansson, S. Jain, J. Montoya-Torres, J. Hugan, and E. Yücesan, 32-39. Piscataway, New Jersey: Institute of Electrical and Electronics Engineers, Inc.

Dorey, S., J. Oehmen, and R. Valerdi. 2012. "Enhancing Cost Realism through Risk-Driven Contracting: Designing Incentive Fees based on Probabilistic Cost Estimates." Defense Acquisition Research Journal 19, no. 2: 133-158. Accessed July 15, 2012. http://www.dau.mil/pubscats/PubsCats/ AR\%20Journal/arj62/Dorey_ARJ62.pdf.

Fuerst, W. 1981. "Small Businesses Get a New Look at ABC Analysis for Inventory Control." Journal of Small Business Management 19 no. 3:39-44. ABI/INFORM Global (Document ID: 66173293).

Ingalls, R. G., M. Cornejo, C. Methapatara, and P. Sittivijan. 2008. "Integrating Simulation and Ooptimization Research into a Graduate Supply Chain Modeling Course." In Proceedings of the 2008 Winter Simulation Conference, edited by S. J. Mason, R. R. Hill, L. Mönch, O. Rose, T. Jefferson, and J. W. Fowler, 2527-2533. Piscataway, New Jersey: Institute of Electrical and Electronics Engineers, Inc.

Law, A. M., and W. D. Kelton. 2000. Simulation Modeling and Analysis. 3rd ed. New York: McGrawHill, Inc.

McKinsey \& Company in conjunction with Georgia Institute of Technology. 2008. The Race for Supply Chain Advantage: Six Practices that Drive Supply Chain Performance. Accessed July 15, 2012. http:/www.mckinsey.com/clientservice/operations/latestthinking/race_for_supply.asp

Robinson, S. 2004. Simulation: The Practice of Model Development and Use. Chichester, West Sussex, England: John Wiley \& Sons.

Rossetti, M. D. and A. V. Achlerkar. 2004. "A Constrained Clustering Algorithm for Spare Parts Segmentation." In Proceedings of the 2004 Industrial Engineering Research Conference, edited by R. King and B. Norman. Houston, TX. Accessed July 15, 2012. http://www.uark.edu/ rossetti/ conference_papers/ierc2004b_achlerkar.pdf.

Rossetti, M. D. and A. V. Achlerkar. 2009. "Evaluation of Segmentation Techniques for Inventory Management in Large Scale Multi-Item Inventory Systems." Accepted for publication in the International 


\section{Bradley and Goentzel}

Journal of Logistics Systems and Management. Accessed July 15, 2012. http://www.uark.edu/ rossetti/journal_papers/ijlsm-achlerkar-paper.pdf.

Sargent, R. G. 2010. "Verification and Validation of Simulation models." In Proceedings of the 2010 Winter Simulation Conference, edited by B. Johansson, S. Jain, J. Montoya-Torres, J. Hugan, and E. Yücesan, 166-183. Piscataway, New Jersey: Institute of Electrical and Electronics Engineers, Inc.

Saylor, S. E., and J. K. Dailey. 2010. "Advanced Logistics Analysis Capabilities Environment." In Proceedings of the 2010 Winter Simulation Conference, edited by B. Johansson, S. Jain, J. MontoyaTorres, J. Hugan, and E. Yücesan, 2138-2149. Piscataway, New Jersey: Institute of Electrical and Electronics Engineers, Inc.

Sherbrooke, C. C. 2004. Optimal Inventory Modeling of Systems 2nd ed. New York: Springer.

Shoaf, S. A. 1983. "A Modular Approach to the Simulation of Manufacturing Processes." In Proceedings of the 1983 Winter Simulation Conference, edited by S. Roberts, J. Banks, B. Schmeiser, 357-362. Piscataway, New Jersey: Institute of Electrical and Electronics Engineers, Inc.

Silver, E. A., D. F. Pyke, R. Peterson. 1998. Inventory Management and Production Planning and Scheduling 3rd ed. New York: John Wiley \& Sons.

Skoogh, A., J. Michaloski, and N. Bengtsson. 2010. "Towards Continuously Updated Simulation Models: Combining Automated Raw Data Collection and Automated Data Processing." In Proceedings of the 2010 Winter Simulation Conference, edited by B. Johansson, S. Jain, J. Montoya-Torres, J. Hugan, and E. Yücesan, 1678-1689. Piscataway, New Jersey: Institute of Electrical and Electronics Engineers, Inc.

Webster, M. D. 2011. Personal communication. December 1. Massachusetts Institute of Technology, Cambridge, MA.

\section{AUTHOR BIOGRAPHIES}

RANDOLPH L. BRADLEY received his Master of Engineering in Logistics and Supply Chain Management at the Massachusetts Institute of Technology, U.S.A., in 2012. As a Technical Fellow within the Supply Chain Management organization of The Boeing Company's Defense, Space \& Security business, his primary role is to enable the transition from successful proposal strategy to flawless program execution, focusing on delivering value across a spectrum of supply chain business opportunities. He is experienced with requirements definition to provide focused solutions, statistical demand forecasting to estimate future requirements, strategic inventory optimization to minimize investment, simulation to reduce risk and predict performance over time, tactical asset management to enable efficient daily operations, and inventory management to warehouse assets. His email address is art2part@alum.mit.edu.

JARROD GOENTZEL is founder and director of the Humanitarian Response Lab at the Massachusetts Institute of Technology, U.S.A., and is a research associate in the MIT Center for Transportation and Logistics. His research focuses on supply chain design and management, transportation procurement and planning, information management and decision support technology. Dr. Goentzel has developed graduate-level courses in supply chain finance, international operations and humanitarian logistics and has extensive experience using simulation games to develop intuition and leadership skills. He also directs the MIT Renewable Energy Delivery project. His email address is goentzel@mit.edu. 